\title{
TIEFE BEINVENENTHROMBOSEN
}

\section{Große Dicke besonders gefährdet}

Adipöse Menschen haben ein erhöhtes Risiko für Thromboembolien. Das Risiko ist auch bei großen Menschen erhöht, fanden jetzt norwegische Forscher heraus. Besonders gefährdet sind große Menschen, die auch noch dick sind. Im Vergleich zu normalgewichtigen, durchschnittlich großen Männern ist das Thromboserisiko bei übergewichtigen Männern $\geq 1,82$ m 5,3-mal höher. Bei normalgewichtigen großen Män- nern ist es um den Faktor 2,6 erhöht und bei kleinen $(\leq 1,62 \mathrm{~m}$ ) dicken Männern um den Faktor 2,1. Bei Frauen $\geq 1,67 \mathrm{~m}$ ist das Risiko bei zusätzlichem Übergewicht um den Faktor 2,8 erhöht. Bei kleinen $(\leq 1,59$ m) dicken Frauen ist der Faktor 1,8. Bei großen normalgewichtigen Frauen zeigt sich keine Risikoerhöhung.

Arterioscler Thromb Vasc Biol, Apr 2011; doi: 10.1161/ ATVBAHA.110.218925

\section{SCHRITTMACHER- UND DEFI-PATIENTEN}

\section{Keine Gefahr am Airport}

Herkömmliche tragbare Metalldetektoren, wie sie bei Sicherheitskontrollen verwendet werden, stellen keine Gefahr für Herzpatienten mit implantierten Schrittmachern, Defibrillatoren oder kombinierten Schrittmacher-Defi-Geräten dar.

Dies ergab eine Studie von Forschern des Deutschen Herzzentrums München und des Dunant Krankenhauses Athen. Sie hatten bei 170 Patienten ( 97 mit Herzschrittmacher, 43 mit implantiertem Defi und 30 mit (RT-ICD) geprüft, ob zwei tragbare Metalldetektoren, wie sie auf Flughäfen eingesetzt werden, Funktion, Batteriestatus oder

\section{ANTIDEPRESSIVE THERAPIE}

\section{NSAR schwächen die SSRI-Wirkung}

Wenn depressive Patienten auf die antidepressive Therapie nicht ansprechen, könnte dies daran liegen, dass sie gleichzeitig nicht steroidale Antiphlogistika (NSAR) einnehmen. In der STAR*D-Studie sprachen depressive Patienten nur zu $40 \%$ auf die antidepressive Therapie mit SSRI an, wenn sie parallel mit NSAR behandelt wurden. Bei Patienten, die kein Antiphlogistikum einnahmen, lag die Rate hingegen bei 54\%.

PNAS online vom 25.4.2001, doi: 10.1073/ pnas. 1104836108

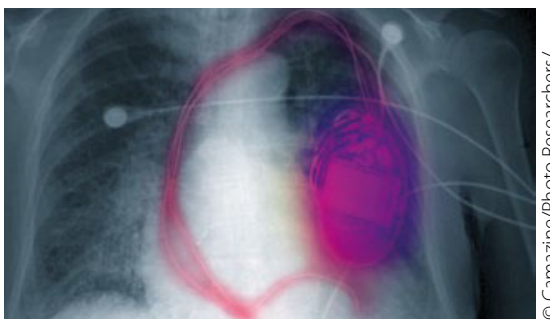

Trotz ICD: Der Sicherheitscheck ist sicher.

gespeicherte Daten der Geräte beeinflussen. Das Ergebnis: Die Magnetfelder betrugen 3,82 und 6,3 Tesla, die Geräte wurden nicht beeinträchtigt.

DGK-Jahrestagung, April 2011; Jilek et al. Abstract P 1577

\section{HERZINFARKT}

\section{Morgenstunden doppelt riskant}

Früh morgens besteht nicht nur das höchste Risiko, einen Herzinfarkt zu erleiden. Infarkte, die sich am Vormittag ereignen, richten auch den größten Schaden an, so das Ergebnis einer spanischen Studie. Patienten mit Beginn eines ST-Hebungsinfarkts in der Zeit zwischen sechs und zwölf Uhr morgens wiesen die höchsten Kreatinkinase- und Troponin-I-Spiegel auf - ein Signal für einen besonders ausgedehnten Infarkt. Heart 2011; doi:10.1136/hrt.2010.212621

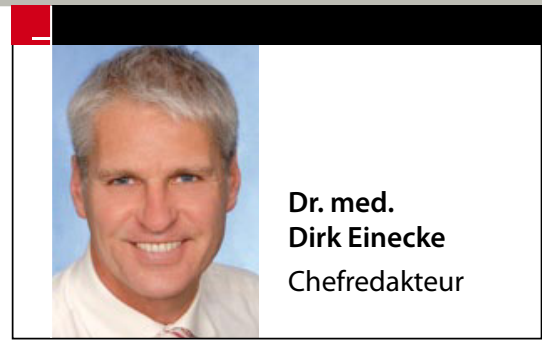

HERZINSUFFIZIENZ

\section{Mündige Patienten leben länger}

Welche Informationen ein Patient über seine Erkrankung hat, wie gut er sie versteht und ob er in der Lage ist, Konsequenzen daraus zu erkennen: All das beeinflusst den Verlauf einer Krankheit. Bei Herzinsuffizienz ist dies sogar ein unabhängiger Prädiktor für die Prognose. Das ist das Ergebnis einer Studie mit 1547 Herzinsuffizienzpatienten, von denen knapp 20\% ein geringes Verständnis ihres gesundheitlichen Zustands attestiert wurde. Bei diesen Patienten war die Mortalität insgesamt deutlich erhöht gegenüber Patienten mit einem adäquaten Wissensstand.

JAMA 2001;305(16):1695-1701
www.SpringerMedizin.de

Highlights vom Internisten- und Kardiologenkongress

Kardiologen aus aller Welt trafen sich vom 27. bis 30. April zur Frühjahrstagung der Deutschen Gesellschaft für Kardiologie (DGK) in Mannheim. Und vom 30. April bis 3. Mai lud die Deutsche Gesellschaft für Innere Medizin (DGIM) zu ihrer Jahrestagung nach Wiesbaden. Die wichtigsten Themen von beiden Kongressen können Sie jetzt schon auf SpringerMedizin.de nachlesen.

www.springermedizin.de/dgk-2011 www.springermedizin.de/dgim-2011 